\title{
An Assessment of Reduced Crew and Single Pilot Operations in Commercial Transport Aircraft Operations
}

\author{
Randall E. Bailey \\ NASA \\ Langley Research Center \\ Hampton, VA \\ randall.e.bailey@nasa.gov
}

\author{
Lynda J. Kramer \\ NASA \\ Langley Research Center \\ Hampton, VA \\ lynda.j.kramer@nasa.gov
}

\author{
Kellie D. Kennedy \\ NASA \\ Langley Research Center \\ Hampton, VA \\ kellie.d.kennedyy@nasa.gov
}

\author{
Chad L. Stephens \\ NASA \\ Langley Research Center \\ Hampton, VA \\ chad.1.stephens@nasa.gov
}

\author{
Timothy J. Etherington \\ Rockwell-Collins \\ Langley Research Center \\ Hampton, VA \\ timothy.j.etherington@nasa.gov
}

\begin{abstract}
Future reduced crew operations or even single pilot operations for commercial airline and on-demand mobility applications are an active area of research. These changes would reduce the human element and thus, threaten the precept that "a well-trained and well-qualified pilot is the critical center point of aircraft systems safety and an integral safety component of the entire commercial aviation system." NASA recently completed a pilot-in-the-loop high fidelity motion simulation study in partnership with the Federal Aviation Administration (FAA) attempting to quantify the pilot's contribution to flight safety during normal flight and in response to aircraft system failures. Crew complement was used as the experiment independent variable in a between-subjects design. These data show significant increases in workload for single pilot operations, compared to two-crew, with subjective assessments of safety and performance being significantly degraded as well. Nonetheless, in all cases, the pilots were able to overcome the failure mode effects in all crew configurations. These data reflect current-day flight deck equipage and help identify the technologies that may improve two-crew operations and/or possibly enable future reduced crew and/or single pilot operations.
\end{abstract}

Keywords - single pilot operations, reduced crew operations, aviation safety, increasingly autonomous systems, automation, automation surprise, crew complement.

\section{INTRODUCTION}

The crew complement - the number of required crew members on-duty on an aircraft flight deck - is established by the design of the aircraft and regulated by design and operating regulations under which it is flown [1]. The number of crew on-board the aircraft may also be augmented because of flight duration and crew duty times. Some long-haul flights require three or four pilots on-board and the duty time on the flight deck, operating the aircraft, is shared to remain within allowable crew duty time limits (see Title 14 of the Code of Federal Regulations, CFR Part 117).

The possibility of reduced crew operations (RCO, i.e., the reduction in the crew complement required to operate a mission) or even single pilot operations (SPO, i.e., operations where only a single human pilot is on-board a flight when more than one human pilot is required today) for commercial airline and on-demand mobility applications is an active area of research. Part of the motivation is that we are witnessing vast improvements in computational power and machine learning that are creating everyday autonomous capabilities such as driving. Many envision $\mathrm{RCO}$, or even SPO, as a next logical step in aviation advancement $[2,3,4,5]$ and often that motivation is economic, either by the reduction of costs associated with employing human pilots or in terms of finding and training enough qualified flight crew for operations. Finally, arguments are also made that the human is increasingly becoming a bottleneck to efficient airspace operations. Machines (i.e., automation) are becoming much more capable and many foresee a future where these machines can perform the requisite aviation tasking and duties as well as if not even better than humans, especially when it is cited that more than $60 \%$ of aviation accidents include human error as a causal factor [6].

The reality is that the change toward RCO or even SPO will require technological innovations and regulatory changes. Quantitative evidence will be required to argue either the pro or con case, where little if any exists today. For instance, often the citation of pilot (human)-error as a causal factor in aviation accidents is used to imply that an improvement in safety can be gained by eliminating the human element on the flight deck. This argument is a complete fallacy. It only argues one side of the problem the forensic analysis. There is no data that identifies how and how often pilots prevent or avert accidents or incidents because these statistics are not kept. Anecdotal evidence, in fact, suggests the opposite of the forensic evidence implying that crews routinely handle and adapt to ever-present and ever-changing operations and system/equipment failures. Airline's Aviation Safety Action Program (ASAP) data [7, 8] supports the importance of the human to safety (although the data are not publicly released). The Air Line Pilots Association argues that "a well-trained and well-qualified pilot is the critical center point of aircraft systems safety and an integral safety component of the entire commercial aviation system." [9] In the case of Part 121 operations, 
highly trained and competent pilots (and well-maintained equipment and proven operations) achieve a level of safety that is estimated to be 10,000 times safer than automobile travel and is often cited as the world's safest mode of travel.

NASA is conducting research to address the needs of future air transportation and airspace operations. Under its strategic plan, NASA is conducting research, development, test, and evaluation to create "assured autonomy for aviation transformation." The Safe Autonomous Systems Operations (SASO) Project is chartered to identify and develop autonomous systems capabilities enabling operational concepts and complexity that sustain significant growth in passengers and cargo beyond the Next Generation Air Transportation System (NextGen). The project's goal is to seek ways to safely integrate within the National Airspace System (NAS) the highest level of autonomy that is justifiable, and develop methods for assurance. NASA is evaluating the efficacy of autonomy technologies that might enable RCO and/or SPO.

This paper describes a research experiment conducted to quantify the human pilot contribution to current-day flight operations. This work helped establish "baseline" levels of performance and safety with nominal crew configuration as well as collect data to assess the performance and safety changes for reduced crew and single pilot crew complements in a present-day flight deck design. The goal is then to identify technology requirements - new automation and more appropriately, increasingly autonomous systems (IAS) - that might assist future twocrew operations and potentially enable RCO or SPO.

\section{BACKGROUND}

The certification requirements for the aircraft's minimum crew are defined under regulations such as 14 CFR Part 25.1523. The minimum flight crew must enable safe operation of the kind envisioned and consider the pilot workload and the accessibility and ease of operation of necessary controls. The basic workload functions to consider are: (1) flight path control; (2) collision avoidance; (3) navigation; (4) communications; (5) operation and monitoring of aircraft engines and systems; and, (6) command decisions.

\section{A. Operational Concepts}

Two conceptually different, but complementary operational concepts are considered herein, in addition to the current-day two-crew complement:

a) Reduced Crew Operations (i.e., single pilot cruise):

In RCO, two human pilots are on-board the aircraft. Two pilots fly, as they normally do so today, during high-workload, congested airspace conditions, such as surface operations, departure, initial climb-out, descent, approach, and landing. However, during the cruise phase of flight, only one pilot is actively engaged in flying the aircraft. The other pilot is resting or possibly napping. (The resting pilot may, in fact, leave the cockpit or may be seated in their cockpit seat.) Augmentation to the crew complement -additional flight pilots/crew, as required today for fatigue mitigation and operational necessity - is not used; instead, alternating rest cycles for the two pilots are employed.

\section{b) Single Pilot Operations (SPO):}

The only pilot on-board the aircraft serves as the captain and pilot-in-command (PIC), making all decisions and performing actions pertaining to command of the flight. In the event that assistance is needed, a ground operator may be linked to the cockpit via digital data-link, video, and/or radio. This assistance may also be scheduled or routinely employed during high-workload conditions such as approach and landing operations. The necessity, extent, timing, security, and expertise required of this remote assistance is the subject of research.

Neither of these concepts are particularly new or novel, with elements covered by patents (e.g., [10], [11]) or similar disclosures [12] as well as significant on-going research being conducted under such programs as the DARPA ALIAS (Aircrew Labor In-Cockpit Automation System) program [13] and the ACROSS (Advanced Cockpit for Reduction Of Stress and Workload) program [14].

In either case, new automation or more aptly, IAS, perform or assist in the functions of the crew member who is now not actively engaged in the flight. The unique, specific challenges for RCO and SPO are described in the following. sections It is felt by some that "the greatest obstacle to the development of a civil, single pilot aircraft is not the technology per se but applying the technology and developing the automation and user interfaces." [3]

\section{B. Remote Pilot Assistance}

NASA has been conducting research into remote pilot assistance, developing various operational paradigms (e.g., "Harbor pilot" or "Super Dispatcher") and most importantly, exploring whether a remote pilot can effectively complete the tasking and reduce the workload to safely enable SPO.

To date, experiments have demonstrated that "separating a two-pilot crew is feasible within the parameters and concepts that were designed and tested." [15] It is assumed that part-time or scheduled, periodic support from a ground operator is a necessary condition for SPO. Thus, a ground operator can handle multiple flights, and if dedicated support is necessary, dedicated assistants can be provided. This concept raises the question of how a ground operator's lack of initial situation awareness (SA), when called on for dedicated assistance, might be an issue. The data shows that, with appropriate displays, ground operators were able to provide immediate assistance, even if they had minimal SA prior to getting a request during en-route scenarios [16]. In a test of SPO during approach to landing, no differences in pilot error rates were shown comparing the nominal, 
continuous flight operation versus the case where the pilot re-entered a flight immediately upon request or after a two minute preparation spool-up time [17]. A primary concern is the ability (or inability) to know what the other pilot is doing [ 18 ]. The design of the ground station, the information, and the security and content of the datalink as well as the expertise of the ground operator are critical issues. In an analogous operational setting, research from the time-critical field of emergency medicine suggests that many modalities of communication must be adhered to for emergency room doctors to quickly and effectively get a team of professionals to create effective medical care. This research [19] showed that visual monitoring of team members' activities was an important part of team coordination and an essential part of team work.

These studies have not fully investigated the value of pilot monitoring [20] for error catching and trapping since it is assumed that these functions will be replaced by IAS technology.

\section{Pilot Incapacitation or Impairment}

In either SPO or RCO concepts, pilot impairment or incapacitation could be catastrophic.

In-flight medical incapacitation is defined as a condition in which a flight crewmember was unable to perform any flight duties and impairment as a condition in which a crewmember could perform limited flight duties, even though performance may have been degraded [21]. Although the data are not definitive [22], ICAO Document 8984 "Manual of Civil Aviation Medicine" employs a "1\% rule" where the risk of incapacitation is assumed to be one percent per annum or approximately equal to one event in 1 million flying hours. Research [23] shows that the incapacitation risk is "small under the age of 40, increases over the age of 50, and rises steeply over the age of 60. ." By age 60 , the data show an average incapacitation rate of approximately $1.2 \%$. Today, the age limit is 65 for Part 121 operations (as of 2009). In-flight incapacitation events are thus predicted to rise with this increase in retirement age, but data are not yet available. Impairment can be as detrimental as incapacitation during RCO or SPO.

As such, the measurement of the pilot's psychophysiological state and identification of adverse human physical and cognitive impairment will be critical technology for RCO/SPO. The development of psychophysiological measures and state identification technologies is on-going, as are appropriate counter-measures [24] to meet the challenges of:

a) Developing sensor suites with appropriate data fusion methods since the results to date suggest that multiple sensor modalities are needed for most classifications;

b) Creating measurement and identification technologies that are robust and reliable enough to match the appropriate performance standards of today's on-board avionics systems (i.e., FAA Advisory Circular AC 25-1309);

c) Meeting these technology goals while simultaneously not over-encumbering the pilot, interfering with their operations on the flight deck, or violating privacy concerns.

This work will also have to include the eventual development of standards for pilot selection and fitness for duty as well as more robust methods for fatigue monitoring and counter-measures.

\section{Fatigue}

The adoption of RCO/SPO may most directly affect the issues of pilot fatigue and the need for attention management.

Under the RCO concept, a regimented period of 'controlled rest' is envisioned as a fatigue counter-measure and critical to the economic viability of the concept. Research supports the contention that "napping is the most effective non-pharmacological technique for restoring alertness." [25] But today, the FAA authorizes in-flight naps for flight crew only if there is an augmented complement so that two pilots are actively engaged on the flight deck while the augmenting crewmembers are resting. A number of foreign air carriers do authorize in-seat cockpit naps. In contrast, SPO implies isolation for the single pilot necessitating, at times, an active means of engagement to prevent boredom and sleep.

Although beneficial for combating fatigue, cockpit napping can have adverse effects because of sleep inertia and other factors [26] [27]. Canadian Aviation Regulations (CAR), described in CAR 2014-1, Part VII - Commercial Air Services, Section 720.23, describe some of the challenges to effectively employ controlled rest, including:

- Pre-flight briefing - planned rest periods can maximize the sleep opportunity and better manage their alertness.

- Pre-rest - the flight crew must prepare for the transfer of duties.

- Rest periods - rest periods should be limited to a maximum of 45 minutes to avoid sleep inertia when the flight crew member is awakened. Rest periods shall occur only during the cruise phase of the flight and shall be completed at least 30 minutes before planned top of descent, workload permitting;

- Post-rest - Unless required due to an abnormal or emergency situation, at least 15 minutes without any flight duties should be provided to the awakened flight crew member to allow sufficient time to become fully awake before resuming normal duties; and (b) an operational briefing shall be given to the awakened flight crew member. 
The methods and processes for removing and reengaging flight crews, in person or remotely, is critical to maintaining effective teaming, situation awareness, and flight crew performance throughout the flight.

\section{E. Increasingly Autonomous Systems}

New automation or more aptly, IAS, must perform or assist in the performance of functions that the second pilot in RCO/SPO flight would normally do. This does not necessarily mean relegating the RCO or SPO pilot to the pilot-monitoring role; the roles and functions for IAS must be tailored to the operation and the needs of the human.

Our work involves the research, development, test, and evaluation of IAS. IAS are envisioned as intelligent machines - hardware and software systems - seamlessly integrated with humans, whereby task performance of the combined system is significantly greater than the individual components. IAS utilize machine learning concepts to perform functions without explicitly being programmed. These systems have the ability to modify their behavior in response to their external environment and conditions. Nevertheless, these IAS are also designed using humanautonomy and automation interaction teaming principals, without the active and latent failings prevalent in many of today's automated cockpits.

IAS, if properly designed, can replicate and in fact should, enhance safety and reliability [28] (e.g., provide the ability to adapt to changing patterns and preferences; remain vigilant at all times; tailor automation actions to specific circumstances/add flexibility; increase situation awareness by context-sensitive information; monitor human actions and alert or intervene to prevent errors; improve automation's ability to react quickly to avoid critical situations). By creating human-autonomy teaming and associated technologies, the unique abilities of intelligent machines and humans are leveraged to create levels of safety and performance above and beyond that provided by either one singularly. Such a system may be especially suited during off-nominal events or in conditions where less experienced or non-expert operators are involved.

\section{BASELINE STUDY}

To kick-off this research toward improving current-day two-crew operations, and potentially enabling SPO/RCO by application of IAS, a baseline study was conducted. The goal was to evaluate and quantify the human pilot's contribution to flight safety during normal flight and in response to aircraft system failures.

A pilot-in-the-loop flight simulation study was performed by the NASA Langley Research Center in partnership with the Federal Aviation Administration (FAA). To quantify the human's contribution, crew complement was used as the experiment independent variable, as follows:
- Nominal two-crew - the Captain (CP) and First Officer (FO) were seated in the left and right seats, respectively.

- Reduced Crew Operation (RCO) - the CP and FO were seated in the left and right seats, respectively, as usual. One pilot was designated as the resting pilot. This pilot remained seated in their seat but, when designated for rest, they donned a visual/audio restriction device to be isolated from the sights and sounds of the flight. The resting pilot returned to active flying duties only after being requested by the pilot-flying, but with experimental constraints imposed to control their reentry to flying duties.

- Single Pilot Operations (SPO) - the CP or FO was seated in the left seat and the other pilot was not in the simulator. Although this is not the usual seat for the FO, this methodology was planned because the left seat has access to all normally required equipage (e.g., the tiller) as would be expected for an aircraft designed for SPO. Further, pilots are required to fly from the left seat to obtain their aircraft type rating so all pilots have trained in the left seat.

The different crew complement conditions were flown in a between-subjects experiment during both nominal and six non-normal scenarios of various severity.

Although NASA's interest is in future technologies, as a baseline study, new technologies were not introduced. The test utilized a present-day flight deck design to quantify the current status of flight deck and airspace operations. These data are fundamental to and critical for the design and development of future IAS that can better support the human in the cockpit.

A brief overview of the experiment is provided in the following with references to other publications as they contain more detailed analysis of the test methods and specific scenario results. This paper focuses on the overall assessment of dual-crew versus RCO or SPO and the technology requirements derived from these data for increasingly autonomous systems that might assist future two-crew operations and eventually possibly enable reduced crew or single pilot operations.

\section{EXPERIMENT}

\section{A. Participants}

Thirty-six pilots (18 crews), representing 5 major US airlines, participated in this experiment. Each pilot held an Airline Transport Pilot rating and was current in the B737800 aircraft as either CP or FO. Crews were paired from the same employer to minimize inter-crew conflicts in Standard Operating Procedures (SOPs) and Crew Resource Management (CRM) procedures or training. All participants were male. 


\section{B. Apparatus}

The research was conducted using the B737-800 simulator operated by the FAA AFS-440 at Oklahoma City, OK. The simulator, although a Level D approved training device, is also fitted with experimental controls, modifications, and recording capability to support AFS440's research mission.

The test was set-up to replicate a normal airline operation and mimic the 'real-world' to the best of our ability. An air carrier flight from Denver (KDEN) to Albuquerque (KABQ) was used. Dispatch paperwork for the flight was developed and used by the crews. A key element of the test was a live controller and various pseudopilots tied into the simulation radio to emulate Air Traffic Control (ATC) for realism and to maintain realistic pilot workload levels.

\section{Independent Variables}

The independent variables were crew complement and scenario. Three (3) crew conditions as described above were used: nominal two-crew, RCO, and SPO in addition to six non-normal scenarios.

\section{Nominal Operations}

The crews were instructed to use their normal company SOPs, their Quick Reference Handbooks (QRH), charts, and checklist procedures for the entire test, including any company dispatch calls and cabin crew communications as SOP. After the crews had reviewed the paperwork and discussed amongst themselves the flight and flight conduct, the crew manned the aircraft (simulator), did a quick familiarization check and reviewed the simulator safety briefing. The crews were instructed to fly the (simulator) flight as if it were a normal revenue flight for their airline. Of particular note, the Auxiliary Power Unit (APU) was out-of-service for the flight. (The APU is not a minimum equipage list item.)

The crew flew an entire "nominal" flight from KDEN to $\mathrm{KABQ}$ following the planned route of flight as their first event in the test. The nominal flight served as a baseline for 'normal' airline operations (i.e., nominal data) and also helped to garner familiarity for the two-person crew interaction. This nominal flight was 1 hour 25 minutes of flight time.

Six (6) nominal RCO flights were also conducted with the $\mathrm{CP}$ as the resting pilot during the cruise leg of the flight and 18 pilots flew nominal SPO runs from Top-of-Descent (TOD) to KABQ.

\section{E. Scenario Categories}

Six (6) non-normal experimental scenarios were designed. The scenarios modulated workload and automation issues (e.g., by the availability of the autopilot after the failure) and flight crew awareness and monitoring during the normal/non-normal operation.
The experimental scenarios started at either the Top-ofClimb (TOC) or approximately 15 minutes prior to the TOD along the nominal flight plan. The simulation was reset to these initial conditions and the crew/pilot were briefed on the starting position prior to going to "operate." These two conditions were used for test expediency. The crews were briefed that the flight to this point was nominal. All flights were flown to completion (i.e., landing).

The test matrix is shown in Table 1 with the number of populated cells $(\mathrm{N})$ defined with the 18 crews:

The six non-normal scenarios have all occurred in revenue service (although, obviously, the exact circumstances are different) and with the 3 crew configurations, stressed all of the pilot workload factors, to various degrees, outlined in Appendix D of 14 CFR Part 25, including: (1) The accessibility, ease, and simplicity of all necessary controls (e.g., the hydraulic leak required manual gear extension); 2) The accessibility and conspicuity of all necessary instruments and failure warning devices. (e.g., the overspeed clacker during the unreliable airspeed failure is an extremely loud and intrusive alarm); 3) The number, urgency, and complexity of operating procedures (e.g., the generator failure stressed systems knowledge); (4) The degree and duration of concentrated mental and physical effort involved in normal operation and in diagnosing and coping with malfunctions and emergencies; 5) The extent of required monitoring of the systems; 6) The degree of automation provided (e.g., the rudder trim runaway required significant manual control activity); 7) The communications and navigation workload (e.g., single pilot operations for the $\mathrm{C}$ category failures are significantly taxing since the autopilot is not available); and, 8) the possibility of increased workload associated with any emergency that may lead to other emergencies (e.g., the loss of both generators was a time critical emergency that could lead to a complete loss of electrical power).

Post-test, pilots were given a questionnaire and additional ratings and comments were taken as described in the following.

\section{RESULTS}

This paper provides an evaluation of crew configuration influence across the entire array of normal and non-normal operations using a current-day flight deck configuration during two-crew, reduced crew, and single pilot operations. Specific review of each non-normal and its specific effects and influences are contained in [29, 30, 31, 32, 33].

\section{A. Objective Performance Data}

The objective performance data are essentially unique for each of the non-normal events because of the vast array of failure conditions and effects. These data, therefore, are not generalizable (see each of the individual papers). There are some observations that are generally indicated:

- Checklist usage is more consistent and accurate in two-crew vs. RCO or SPO operations. In RCO, the 
start of the checklist was often delayed until the resting pilot came back to flying duties. The RCO condition was also occasionally problematic since the flying pilot may or may not have started the appropriate checklists. If the crew did not do a thorough 'return-to-duty' briefing, the returning pilot may have assumed that the checklists were done, when in fact, they were not. A specific "pre-rest" briefing checklist was imposed in the experiment but a "return to duty" briefing was not. This was left to the crews and is similar to current long-haul flights that use augmented crews. In SPO, significant task shedding occurred and checklist usage was often sacrificed. The difficulty of hand-flying and troubleshooting by a single pilot created complications in getting to the checklist and it encouraged troubleshooting outside of the checklist.

- Flight path performance was better in two-crew than SPO or RCO. Two pilots provided for a pilot monitoring. Two-crew operations provided four hands, four eyes, and two brains to monitor and work the problem(s). Two pilots provided for workload sharing, especially in the rudder trim runaway nonnormal where the physical workload to control the vehicle was significant. Two pilots provided a larger wealth of experience from which to draw from such as adapting to unique flying techniques (using asymmetric thrust to help balance fuel load or rudder trim), knowing nearby available, suitable, but underused airports (Grand Junction, $\mathrm{CO}$ ), or understanding secondary or compounding failure effects that are not checklist items (loss of generators will cause pressurization effects).

- To date, we have not quantified the pilot errors; that work is on-going. In general, more errors were committed in SPO than RCO, but errors were committed in all crew conditions. Errors of omission and commission were observed.

\section{B. Pilot Workload-Nominal Flight}

Observations from the test suggest that the primary determinant of safety of flight and the propensity for flight crew errors was directly related to pilot workload. When the pilot workload exceeded certain limits, the pilots would shed tasks and in doing so, errors in execution or omission would occur.

The NASA Task Load Index (TLX) [34] captured a subjective rating $(0-100)$ of perceived task load. There are six subscales of workload represented in the TLX: mental demand, physical demand, temporal demand, performance, effort, and frustration. The overall and subscale score results of this measure were examined to investigate task load variation.

The Captain's (CP) workload for the three crew configurations, using the average TLX rating across the 6 components, is shown in Figure 1. The box plot data of Figure 1 and in the subsequent figures show the mean value by the red line, the $25^{\text {th }}$ and $75^{\text {th }}$ percentile by the extent of the box, with the 5\% statistical spread from the mean shown by the indent. The whiskers show the maximum and minimum values up to $1.5 \mathrm{x}$ the interquartile range. Plus signs indicate ratings outside of $1.5 \mathrm{x}$ interquartile range.

Interestingly, the workload for the CPs is reduced in the RCO configuration compared to the two-crew configuration during nominal flight conditions. These data are anomalous as there are two effects in play. First, RCO means the Captain had a rest period. Crews noted that the RCO concept, where one of the pilots is resting in the cockpit (napping), can be an effective fatigue mitigation. This fact is resident in these data and it has also been an observation supported by other research as previously reviewed.

Table 1. Experimental Test Matrix

\begin{tabular}{|c|c|c|c|c|c|}
\hline \multirow{3}{*}{\multicolumn{2}{|c|}{ Scenarios }} & \multicolumn{4}{|c|}{ Crew Complement } \\
\hline & & \multirow{2}{*}{$\begin{array}{c}\text { Nominal } \\
\text { Two }\end{array}$} & \multicolumn{2}{|c|}{ RCO } & \multirow[t]{2}{*}{ SPO } \\
\hline & & & FO Resting & CP Resting & \\
\hline & Nominal & 18 & 3 & 6 & 18 \\
\hline \multirow[t]{2}{*}{ A } & Unreliable Airspeed & 6 & 3 & 6 & 6 \\
\hline & Engine Fuel Leak & 6 & 3 & 6 & 6 \\
\hline \multirow[t]{2}{*}{ B } & Reservoir Hydraulic Leak & 6 & 3 & 6 & 6 \\
\hline & Generator Drive Shaft Failure & 6 & 3 & 6 & 6 \\
\hline \multirow[t]{2}{*}{$\mathrm{C}$} & Loss of Both Generators & 6 & 3 & 6 & 6 \\
\hline & Rudder Trim Runaway & 6 & 3 & 6 & 6 \\
\hline
\end{tabular}


Secondly, the experiment design artificially introduced biased (higher) workload ratings for the nominal two-crew condition. The nominal two-crew was run as the first event for the subjects and this run was not repeated. In hindsight, the experiment should have used the first run as familiarity and thrown the data away. In the first run, the subjects naturally have some anxiety, being in a new environment and being subjects in a research experiment. As such, their self-assessed workload skewed high.

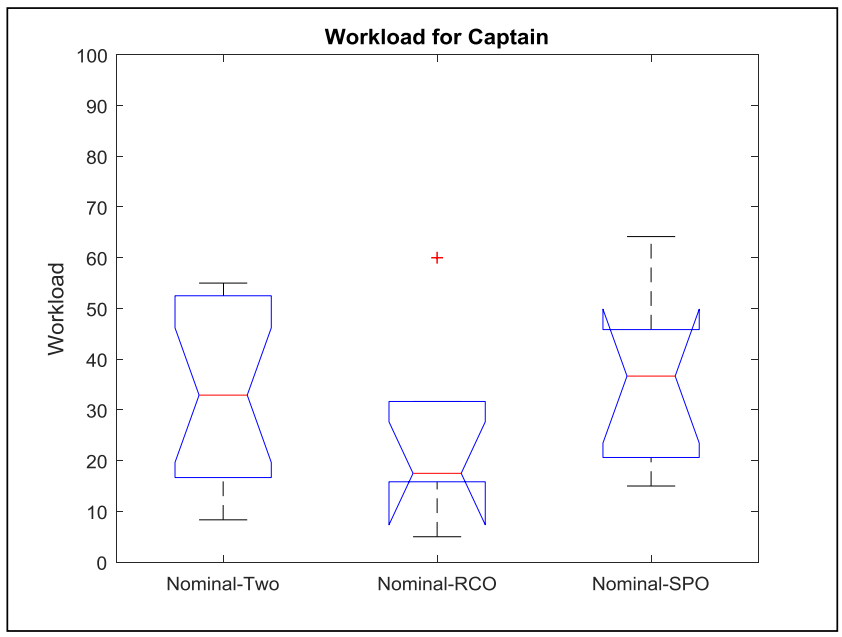

Fig 1. CP TLX Workload Rating by Crew Configurations - Nominal Flight

Workload for the Captain in the SPO condition trends much higher than the RCO condition, but not tremendously so. The mean rating for nominal SPO was only 37 out of 100 maximum.

The data for the FO shows similar trends as the CP but to greater extremes (Figure 2). The RCO condition is significantly lower in workload than the two-crew configuration and the workload in the SPO configuration for the FO is significantly higher than RCO and higher than for the Captain.

The same workload anomaly for the two-crew configuration for the CPs is reflected in the FOs as well. The two-crew workload is anomalously high. In the case of the RCO condition, the low workload reflects a nominal flight condition - so the FO didn't have much to do and was single pilot only during the cruise phase of flight - and that the workload for the descent, approach and landing for the FO was less overall workload since the $\mathrm{CP}$ was now rested and always took the last flight leg. The trend for higher workload by the FO during SPO is likely due to a CP's greater experience and familiarity with being responsible for the entire operation. Further, by experiment design, we put the FO in the left seat; thus, increasing their workload to acclimate to a different seat.

\section{Pilot Workload-Non-Normals}

In the presence of non-normals, the workload data from the $\mathrm{CP}$ and $\mathrm{FO}$ became almost identical. The workload data across all non-normal scenarios is collapsed across pilots and is shown in Figure 3. The data show a statistically significant increase in workload for the SPO condition compared to the nominal two-crew and RCO configurations (which did not statistically differ from each other). The data also reflects the wide disparity of non-normals tested, from the relatively benign hydraulic leak to the rudder trim runaway and dual generator failures which created significant physical and temporal workload, respectively. The data extremes are shown in the whiskers of the box plots.

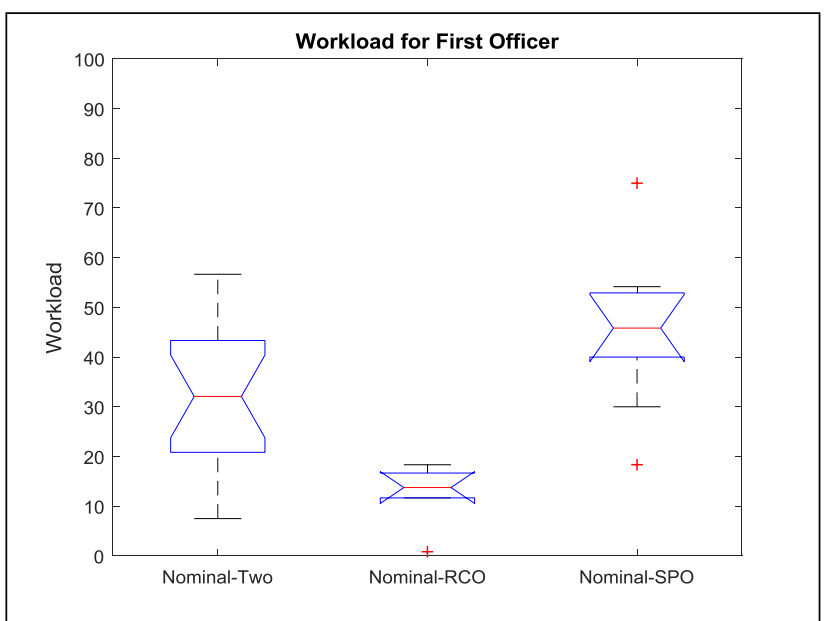

Fig. 2. FO TLX Workload Rating by Crew Configurations - Nominal

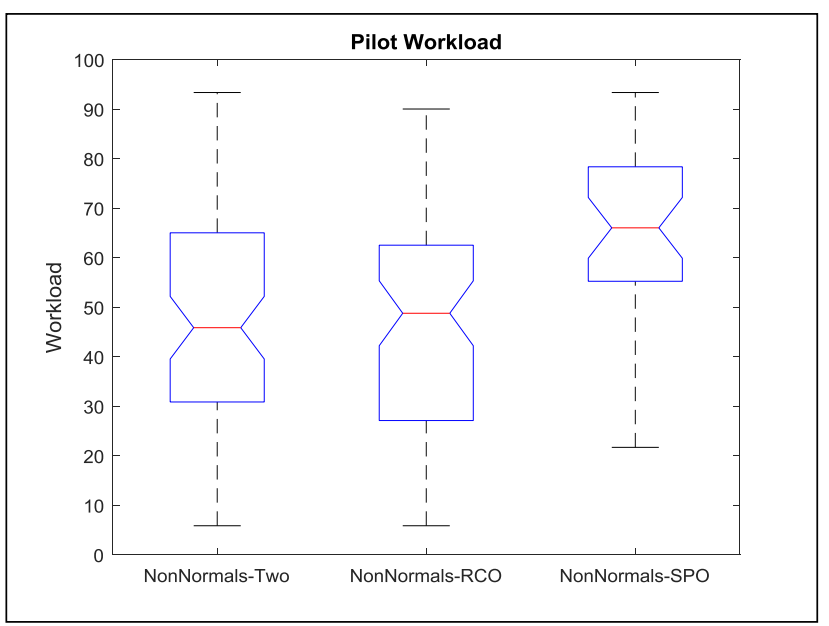

Figure 3: Pilot Workload for Non-Normal Events

Although the workload data shows that SPO is not desirable from a workload standpoint, all crews/pilots were 
able to safety conduct and complete a safe landing. Significant task shedding was noted, especially in the case of checklists. Errors and procedural omissions were noted, although errors and procedural omissions were also noted during two-crew and RCO conditions. The TLX physical demand data (not shown) is indicative of how the change in the crew complement significantly impacts the number of switch actions, button presses, control inputs, and other ancillary duties which are now the burden of a single pilot; these changes significantly increasing the risk of errors on the flight deck.

\section{Post-Test Workload Comparison}

A post-test, paired comparison was conducted with the subjects for subjective workload assessment of the three crew configurations. The post-test comparisons, using the Subjective Workload Dominance (SWORD) [35] test, quantified the relative workload across the three crew configurations (Two-crew, RCO, SPO) while dealing with a non-normal event. The $\mathrm{CP}$ and FO independently completed the SWORD comparisons. For these comparisons, workload was defined as "the integrated physical and mental effort to perform the assigned task."

Independent Analysis-of-Variance (ANOVA) revealed crew configuration was significant for CP SWORD ratings $(\mathrm{F}(2,51)=116.39, \mathrm{p}<0.0001)$ and $\mathrm{FO}$ SWORD ratings $(\mathrm{F}(2,51)=93.51, \mathrm{p}<0.0001)$. Post-hoc tests (SNK using $\alpha=0.05$ ) showed the same three unique subsets for the CP and FO SWORD ratings: 1) Two-crew (lowest workload), 2) RCO, and 3) SPO.

These data confirmed that crew complement significantly influenced pilot workload.

\section{E. Crew Resource Management (CRM)}

The effect of RCO on Crew Resource Management was subjectively self-rated by the $\mathrm{CP}$ and $\mathrm{FO}$ using a 7 point Likert scale from strongly agree (1) to strongly disagree (7) in response to five (5) questions related to the indices of CRM (i.e., from AC120-51E, Section 14(a)), focused on: a) communication; b) decision-making; c) team-building: d) workload maintenance; and, e) SA. These data were collected following the completion of the two-crew and $\mathrm{RCO}$ evaluations.

These subjective data show no significant differences in ratings between the $\mathrm{CP}$ and $\mathrm{FO}$ or between the two-crew and RCO operation. The crews did not feel that RCO impacted CRM.

To quantify the "reengagement" by the resting pilot, the pilots were asked two questions using a seven-point Likerttype scale to gauge the timing and effectiveness of the resting pilot re-engagement back into the simulation. The first question was whether the time the resting pilot reentered the flight was "too early" (rating of 1) vs. "too late" (a rating of 7) with a rating of 4 being "just right." The second question asked whether the reengagement was "completely ineffective" (rating of 1) versus "completely effective" (rating of 7) and a rating of 4 ("neutral"). These data are shown in Figure 4 and 5.

The data in Figure 4 show that the reengagement by the CPs was sometimes felt to be too late even in the nominal case, whereas the FO thought the time was fine in nominal flight conditions. (We could not follow the CAR recommendations due to flight time limitations for our experimental scenarios; the resting pilot, $\mathrm{CP}$, was brought back to duty just prior to TOD.) These responses might suggest that the CPs experienced some elements of rest/fatigue or sleep inertia and wanted to return to duties earlier to be more awake and aware. Both pilots agreed that it was generally later than desired during non-normal events, but not universally so. (One $\mathrm{CP}$, in fact, rated the time as "too early." In this case, this rating was given because he was "engaged" too early in returning to duty. He felt that he needed to come up to speed with the situation before he started making decisions and performing actions.)

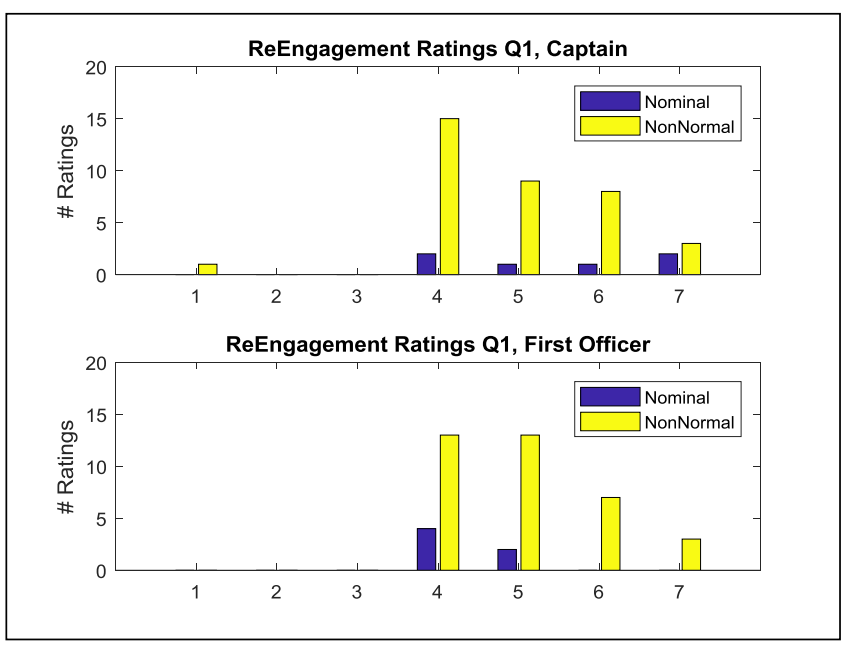

Figure 4. Resting Pilot Re-Engagement Ratings

The data for effectiveness of the resting pilot's return shows that, in general, the return was effective, if not completely effective. The assessments by the CP and FO were nearly identical in this regard. The crews subjectively felt that the rest period did not impact their effectiveness.

These results are not really surprising. The crews were paired by airline so SOP differences were minimized and effective teaming is part of their organizational structure (for the airlines tested). For RCO, the experiment team developed and trained a 'before rest' crew checklist that defined how to prepare for the rest period and under what conditions the resting pilot was to be awaken. There were only 2 minutes when the crews were separated after the flying pilot requested the resting pilot to return; although this can be a significant duration when trying to handle a failure as a single pilot, it did not destroy the CRM - all crews, once the CP came back into their flying duties, started to actively communicate the situation (i.e., the FO briefed the $\mathrm{CP}$ what happened while they were sleeping) and 
the two pilots started verbally coordinating tasking that each would perform to address the failure and its consequences. Their communication and actions were not always perfect, but there was evidence of active CRM in all RCO conditions. The crews thought this was between neutral and completely effective.

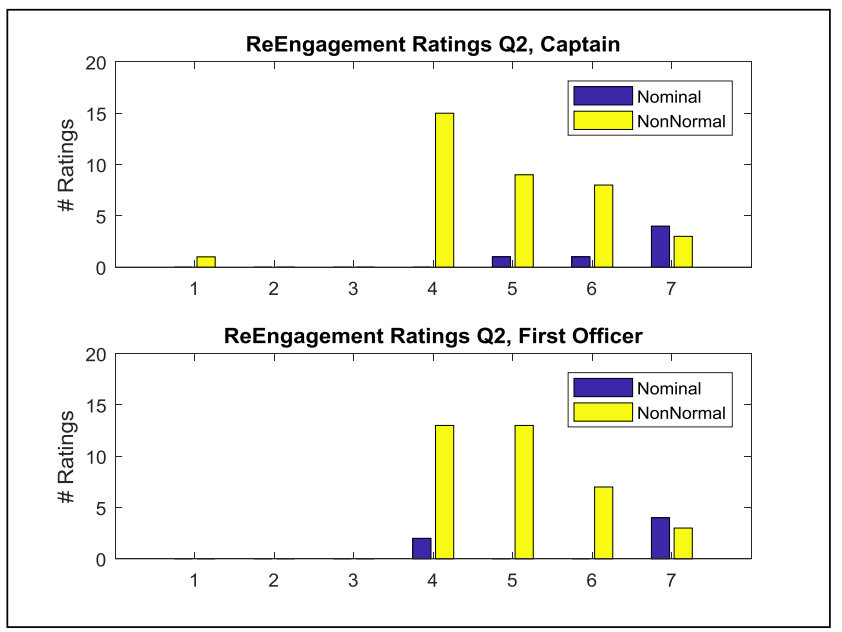

Figure 5. Resting Pilot Re-Engagement Ratings

Objective data and PI observations found that the postrest briefing for RCO is very important. The test did not create a 'post-rest' crew briefing checklist, but instead, left it unstructured. The resting pilot does not have a shared experience of the event and how the resting pilot can come up to speed with what they missed can be invaluable. If all the critical elements are not briefed, the resting pilot will often go down the wrong path or make erroneous assumptions. In some cases, the data showed missing checklist items because the crews did not fully brief each other on their reengagement. For instance, in the case of the unreliable airspeed non-normal, if the flying crew member already returned the aircraft back to the cruise altitude before the resting pilot was engaged, the failure indications were no longer present. The resting pilot might not have been aware of any problem since all symptoms would have been extinguished.

\section{F. Perceived Safety (Post-Run)}

Perceived safety was assessed after each run using a seven point Likert scale with a rating of 1 being "completely acceptable," a rating of 7 being "completely unacceptable," and a rating of 4 being "neutral." The data did not show any significant differences or trends in the rating data by the $\mathrm{CP}$ or FO. The perceived safety ratings for the pilots (collapsing across $\mathrm{CP}$ and FO) are shown in Figure 6, indicating the effect of crew configuration and nominal vs. non-normal flight conditions.

For nominal flight conditions, the two-crew and RCO configurations are completely acceptable or very near so. Ratings of 'unacceptable' were given for nominal SPO. These data reflect the possibly precarious situation that SPO presents (i.e., in the event of failures, SPO means only one pilot on-board to handle failures) and also, that only one pilot is on-board to monitor operations.

In the event of failures and non-normal conditions, safety is impacted but still preserved during two-crew operations (although some failure conditions trend toward unacceptable conditions, e.g., dual generator failure and rudder trim runaway). The perceived safety for RCO and SPO is unacceptable in the event of failures or non-normal conditions. The RCO configuration data indicates that the tested non-normal conditions compromised safety and, in general, were not acceptable. During non-normal operations, the SPO condition - in general - is completely unacceptable (although for some reasons, some pilots gave 'acceptable' ratings for a few failure events.)
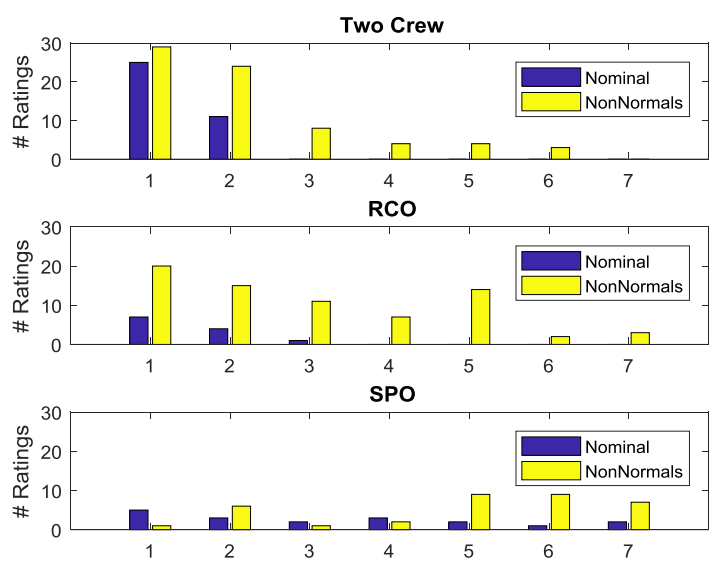

Figure 6: Perceived Safety, Post-Run

\section{G. Failure Effects}

The Failure/Recovery Rating Scale [36] subjectively quantified two events, Effect of Failure and Ability to Recover, using a decision tree - with ratings from $\mathrm{A}$ to $\mathrm{H}-$ that reflects the pilot's perception of the effect of the failure and their ability to recover, respectively. The first rating "Effect of Failure" - ranged from "minimal excursions in aircraft state" (rating of A) to "catastrophic encounter with obstacles or structural failure" (rating of $\mathrm{H}$ ). The second rating - "Ability to Recover" - ranged from "corrective control action not require" (rating of A) to "no possibility of averting catastrophe" (rating of $\mathrm{H}$ ).

The failure/recovery rating data are collapsed across $\mathrm{CP} / \mathrm{FO}$ - they did not significantly differ in their assessments. In Figure 7, the data are shown for the twocrew, RCO, and SPO crew configurations. The data indicate that the two-crew and RCO conditions are not significantly different. There is a slight trend toward worse ratings for the RCO, but nothing dramatically different. On the contrary, the SPO ratings show that even relatively minor nonnormals create notable failure effects and decrements in the ability of the single pilot to recover the aircraft. Ratings of 
$\mathrm{F}$ or worse indicate that the pilots view safety of flight as being compromised. SPO more often creates cases where this happens (Note - there are half the number of ratings for $\mathrm{SPO}$ in these figures compared to two-crew and RCO since there are two pilot ratings for the two-crew and RCO evaluations versus just one for SPO).

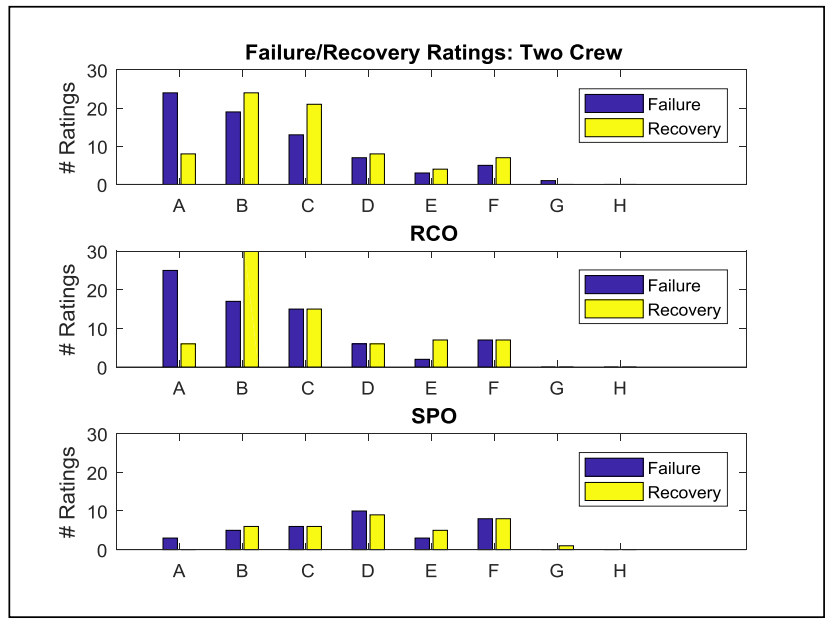

Fig. 7, Failure/Recovery Ratings

\section{H. Post-Test Safety and Acceptance}

Post-test data was used to gather a perspective view from the flight crew of safety and acceptance for RCO and SPO:

- The safety of flight for the RCO and SPO crew complement was evaluated in comparison to current-day two-crew operations using the color-coded, thirteenpoint Likert-type scale (Fig. 8). The scale ranges from "no safety effect" to "catastrophic" safety effects for $\mathrm{RCO}$ and SPO.

- The pilots also used a seven-point Likert-type scale to rate the "acceptability" of RCO and SPO compared to two-crew operations, with a rating of 1 being "completely acceptable" and a rating of 7 being "completely unacceptable."

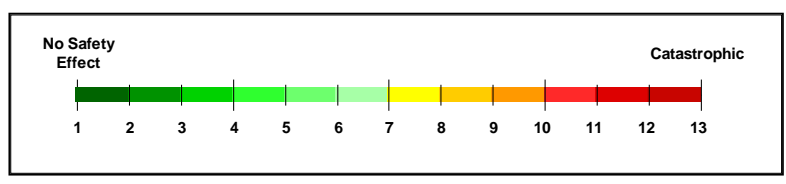

Fig. 8, Likert-Type Rating Scale for Safety Effect

The perceived safety results show that RCO was rated across the spectrum by the pilots for safety and acceptability (see Figure 9 for safety; the acceptability data were analogous). There were no appreciable differences from the CPs vs. FOs. The comments reflect the wide spectrum of rating data - as one noted, the "time delay for crew member to return to his seat has some risk exposure. But not much." And "as long as a secure system is in place to recall the crew member back in matter of max $90 \mathrm{sec}$. It would not be an issue." On the other hand, many other suggested that it is "always better to have two sets of eyes/ brains evaluating from onset of situation." The objective data showed that pilot monitoring is critical to error catching and trapping.

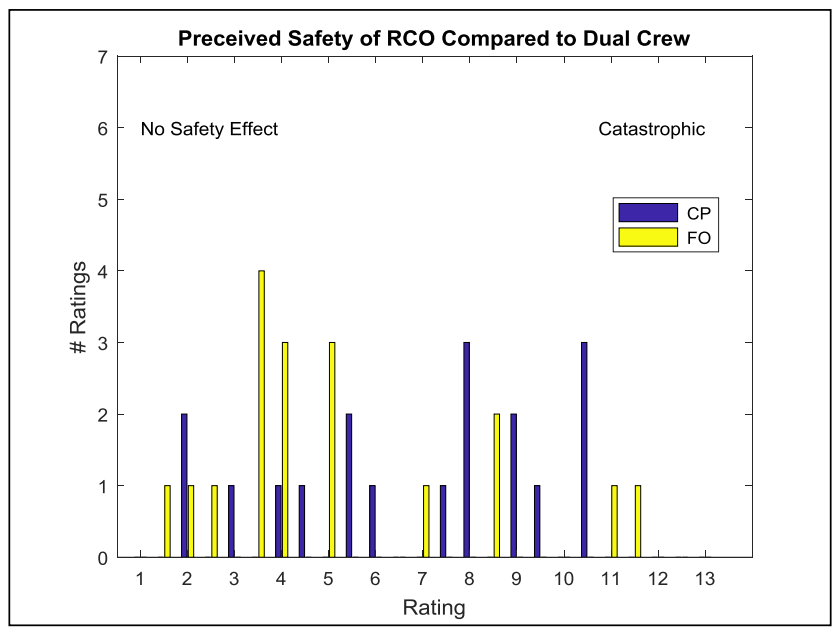

Figure 9: Perceived Safety of RCO compared to Dual Crew

The SPO condition was clearly not well appreciated by the flight crew (perceived safety shown in Figure 10 and acceptability in Figure 11). Some of the best ratings were quantified, "single pilot can be conducted safely except certain non-normal operations could become very risky" but the majority of the ratings reflect the opinion that SPO is "dangerous; no one to share workload or responsibilities." "As the system stands it would be catastrophic - would require vast changes to A/C and ATC system - perhaps impossible - no human safety net - reduces safety and increases risks - environment demands two person effort."

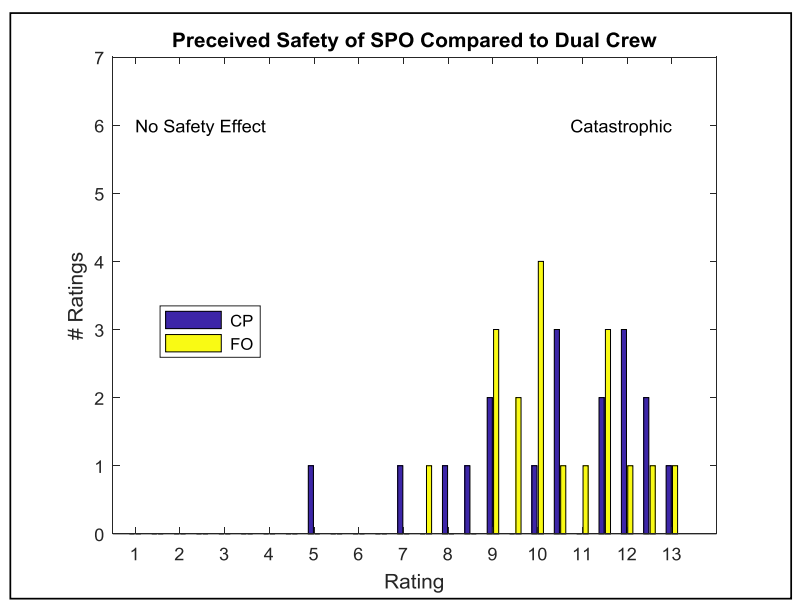

Figure 10: Perceived Safety of SPO compared to Two- Crew 


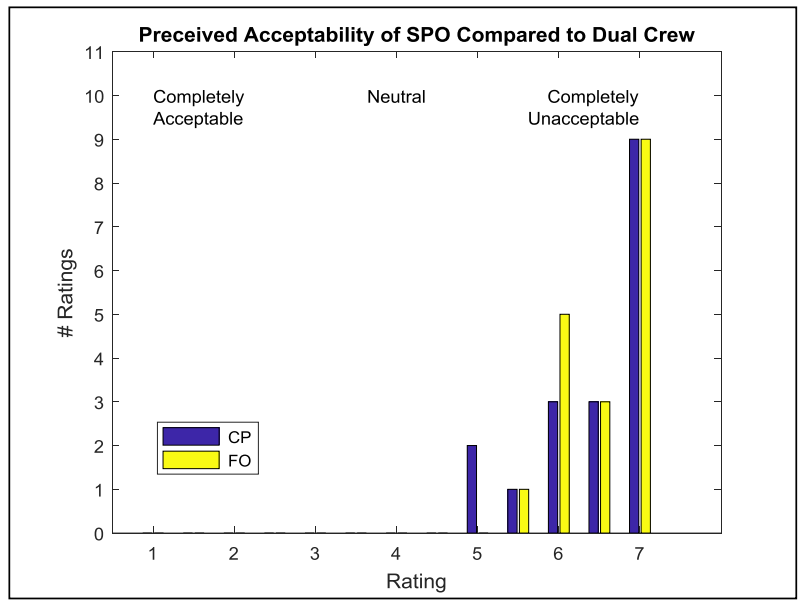

Figure 11: Perceived Acceptability of SPO compared to Two- Crew

\section{TECHNOLOGY}

The test used current-day flight deck equipage. As such, there were limitations for the subjects' assessments of RCO and SPO. However, these data highlighted what technologies are needed to support present-day two-crew operations and if RCO and/or SPO would ever be acceptable in commercial transport aircraft operations.

\section{A. Virtual Co-Pilot Concept}

The SPO condition experienced by the subjects did not include a 'virtual co-pilot' or specialized ground assistant. A virtual co-pilot would be a system/process where a qualified person, e.g., an appropriately trained individual (including but not necessarily limited to a pilot) utilized real-time, two-way transmission of voice and/or text and flight-critical data between an aircraft and a ground-based computer workstation, who could perform a number of functions that would enhance or maintain flight safety such as assisting in the performance of routine checklists, monitoring and communicating with air traffic control, advising the pilot in the aircraft on how to handle any inflight situations that may arise, and transmitting flight routing via Aircraft Addressing and Reporting System (ACARS) or similar data-link for auto-loading into the flight management system.

We used a second post-test workload comparison assessment (using SWORD) to quantify the pilot's assessment of the relative workload ratings while conducting descent, approach, and landing operations for three crew conditions: Two-Crew, SPO, and a Single Pilot with Virtual Co-Pilot concept. The two-crew and SPO were flown in the test.

Independent ANOVA analyses revealed crew configuration was significant for CP SWORD ratings $(\mathrm{F}(2,51)=36.47, \mathrm{p}<0.0001)$ and $\mathrm{FO}$ SWORD ratings $(\mathrm{F}(2,51)=31.08, \mathrm{p}<0.0001)$. Post-hoc tests (SNK using $\alpha=0.05$ ) showed the same three unique subsets for the $C P$ and FO SWORD ratings: 1) Two-Crew (lowest workload),
2) Single Pilot with Virtual Co-Pilot, and 3) SPO (highest workload) while conducting descent, approach, and landing operations.

The Virtual co-pilot as a concept was shown to have merit but flight crews noted several observations:

- The post-test pilot assessments included Likert-type ratings as to whether a "virtual co-pilot concept" would be beneficial and/or make SPO acceptable (note SPO's Unacceptable ratings in Figure 11). The data showed better acceptability of SPO with the Virtual Co-plot (Figure 12) than without (Figure 10). But a wide variety of opinions were provided from "lots of potential ... except maybe for incapacitation (or) complete ... data transmission loss" to "for nominal flights, sheer boredom would put safety effect in 8-9 range. System failure with no assistance causing manual flight in degraded modes would probably be catastrophic."

- In the experiment, the virtual co-pilot was not simulated per se, but the airline dispatch communications were felt by the flight crew to be a type of virtual co-pilot. Dispatch provided some support, depending upon the flight crew requests. Some asked for checklist support whereas others did not. Many thought that if the "virtual pilot doesn't have all the parameters of flight available to him the single pilot would be spending most of his time relaying environmental issues." Most flight crews during SPO did not use dispatch because they could not afford the time, workload, and communications bandwidth to try to get dispatch up-to-speed with the problem to the extent that they can be useful.

- All flight deck functions and indications need to be made available and controllable by a human on the flight deck or via remote operation. First-and-foremost, remote control of all functions would be needed in the event of pilot incapacitation for safety and in compliance with regulations.

- The remote pilot would need a direct view or replication of the cockpit. Many crews/pilots noted that if they needed to brief the remote co-pilot on what was happening, they would lose valuable time and increased their workload to the extent that it would kill any benefit of remote assistance. CRM and task coordination would need to be further explored.

- Although some data suggests that remote pilots can come up to speed quickly, these data and others suggest that verbal and non-verbal data are important to CRM. Real-time video as a minimum would be necessary, but it is not clear that it would be sufficient. The crews highlighted that it must be self-evident of who is controlling what switch, doing what task, etc. 
- Overall, however, the pilot sentiments were that "nothing can replace a pilot in the cockpit" and "SPO has no place in (Part) 121 ops."

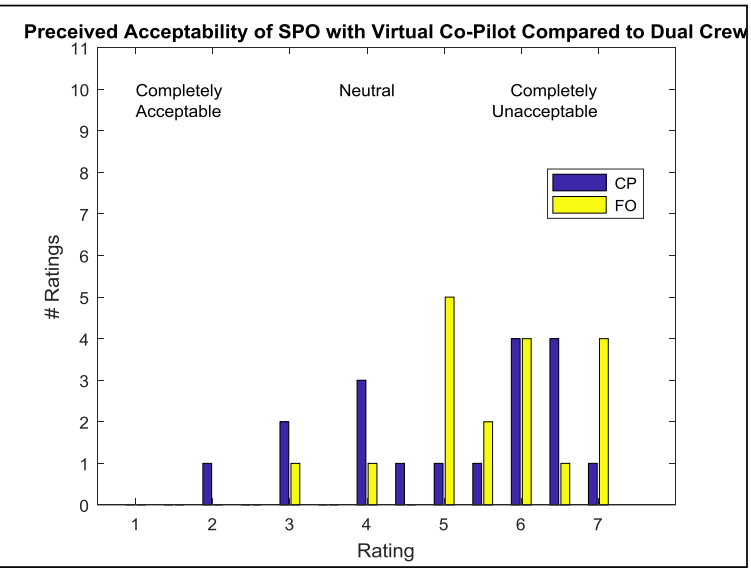

Figure 12: Acceptability of SPO with Virtual Co-Pilot

\section{B. Near-term}

Some technology needs were identified that can clearly support present-day two-crew operations. The items most often noted were:

- The current-day B737-800 Engine Indication and Crew Alerting System (EICAS) and QRH provide awareness of failures and methods to maintain safety-of-flight. However, in many cases flown here, electrical and air system synoptic pages would significantly aid the flight crew in identifying critical items and function elements that had failed. The current processes do not articulate or annunciate the root cause nor the systems and capabilities that are impacted, degraded, or inoperative. Current-day methods rely on the knowledge and skill of the crew to be able to absorb the Caution, Alerting, Warning System (CAWS) data and identify the rootcause. Pilots follow the QRH to 'safe' the system but the current-day SOPs are reactive and significantly relies on expert users (pilots) to stay ahead of situations.

- The current-day CAWS does not often identify what can't be done operationally after the failure. These data should be explicit and not subject to the crew's initiative to dig though the QRH or rely on their experience and expertise [37]. There are now some failures that are annunciated as to what functions are no longer available (e.g., "NO AUTOLAND") but in this test, operations that are not authorized were flown either because the crew didn't have the time, or didn't know that they should dig through the QRH to find the limitations after some failures.

- The crew often cited that they would like automatic, interactive electronic checklists (prioritized from most critical to least) to attend to the failures with speech recognition interfaces to trigger actions.
- The dual generator failure highlighted the need for 60 minute batteries or a Ram-Air Turbine (RAT) electrical source. Today, some airlines equip the B737-800 with only 30 minutes of batteries. Our data suggests that this may not be enough time during adverse weather and remote flight locations.

- The crews were very efficient at donning and configuring oxygen masks but valuable time was lost. This was one of the items often load-shed by the crews (i.e., in their assessment, hypoxia was not going to be a significant concern and they needed the time to attend to other, more critical duties). Automated methods for intercom integration into the oxygen masks would save valuable seconds.

- In augmented crew flying, scripted briefings/checklists for 'before rest' and 'after rest' should be mandated to ensure situation awareness and good CRM upon returnto-duties.

- The possibility of single pilot operations must always be considered a possibility within the current two-crew complement due to pilot incapacitation. The SPO data showed that if the QRH is moved to an Electronic Flight Bag, in addition to charts that already have migrated there, problems will ensue. The single pilot could not easily or effectively use one device for two critical functions. Approval of electronic QRH should be evaluated for this possibility.

\section{Future}

The viability of $\mathrm{RCO}$ and SPO are predicated, as a minimum, on developing IAS for the following tasks:

- First and foremost, SPO and even RCO cannot happen without effective and reliable monitoring of the pilot flying for possible impairment and incapacitation.

- SPO and RCO requires IAS to perform various functions on the flight deck, first-and-foremost being a pilot-monitoring function. The experiment objective data shows that two sets of eyes are better than one. Monitoring of the flight is critical, including decisions, switch actions, and flight path.

- $\mathrm{RCO}$ as a concept has benefit if the incapacitation and impairment challenge can be met and if effective rest can be obtained, without sleep inertia impacts, to combat fatigue. In our experiment, we had one instance where a pilot responded that he actually fell asleep, without detrimental impact in performance.

- The present-day automation design paradigm is not sufficient for SPO/RCO. Current-day automation is strong, silent, and yet brittle. It forces the pilot into the pilot-monitoring role for which the human is not ideally suited, especially if they are the only human on the flight deck. This role would make a difficult task much worse. Autonomy, as opposed to automation, should be robust, responsive to a delegation of tasks and goals 
from the pilot, and adaptive and adaptable to the needs of the human. Autonomy must effectively team with the human to keep them in the loop and situation aware. The communication modalities that pilots use today on the flight deck - natural language, gesture, posture, eye gaze, etc. - must be inherent to the autonomy interface for effective communication.

- The next level for crew state monitoring - above and beyond impairment and incapacitation monitoring and detection - is bi-directional communication with autonomous systems. Bi-directional communication is an essential element for good teaming, including human-autonomy teaming. The team work demonstrated during RCO and two-crew operations was critical to mission success. These teaming principles must be replicated with autonomy.

- A critical element of autonomy support would be monitoring of decisions. Previous work in decisionsupport aids has shown tendencies toward automation bias; a different approach such as the "curious partner" [38] might alleviate confirmation bias. The "curious partner" approach would place the human in the 'proper' role for maintaining SA and expertise, while using the oversight by an autonomous agent to create error checking and trapping. Decision-making support would also need to expand to higher levels of abstraction, answering questions such as "should I?" instead of the question usually answered today: "Can I?"

The development of future technology directions based on this research should not be considered all inclusive; other works, such as [39], highlight some similar, yet different perspectives. Additional research and development will be required, in addition to significant regulatory and societal change, for RCO or SPO to ever become a reality.

\section{CONCLUDING REMARKS}

This experiment evaluated the human pilot contribution to flight safety. Crew complement was experimentally changed to RCO or SPO from the nominal two-crew in an assessment of the level of the safety and performance in today's airspace with today's cockpit design.

The data supports the criticality of the human's role and the adaptability of human pilots/flight crew that is instrumental in overcoming non-normal conditions and in completing safe recoveries, even in SPO. The pilots were able to workload-shed tasks and duties, enlist help from ATC or their airline dispatch center, and perform actions and make the proper decisions in enough time to safely complete the flight within acceptable flight performance limits. The data also indicate, however, that single pilot operations are not nominally acceptable due to the significant task demands and workload. The pilots could overcome the circumstances presented, but rated the workload, safety, and acceptability as being unacceptable in an emergency situation. There were notable flight performance decrements during SPO compared to two-crew operations that suggest unacceptable, reduced safety margins.

Present-day attitudes from the participants were not entirely negative toward RCO. The value of RCO operations was seen to combat fatigue. However, there are identified technology and regulatory hurdles for RCO, in particular, the inertia due to sleep or rest for the resting pilot in terms of how quickly and effectively they can come back to full effectiveness. The resting pilot noted these deficiencies more so than the pilot-flying. In addition, pilot monitoring of the pilot flying for incapacitation and impairment would be critical as well as monitoring of the actions of the pilot flying.

Autonomy to enable RCO and SPO is required; but "more automation" analogous to present-day autoflight systems was not advocated. New technologies increasingly autonomous systems - with new functionalities, responsibilities, effective human-autonomy teaming, using natural language and gestures for example, and robustness are needed.

It is felt by some that "the greatest obstacle to the development of a civil, single pilot aircraft is not the technology per se but applying the technology and developing the automation and user interfaces." These data suggest that while it may be true that new technologies are not needed, we don't have the knowledge to effectively employ these technologies, nor to create the functionality that would be required with the requisite reliability and robustness, as well as the adaptability and predictability to safety implement SPO and RCO today. Additional research and development is necessary.

\section{ACKNOWLEDGEMENTS}

This work was sponsored by NASA's Airspace Operations and Safety Program, Safe Autonomous Systems Operations project, led by Parimal Kopardekar, Project Manager, and Sharon Graves, deputy Project Manager.

This work was conducted as part of an Interagency Agreement, No. IA1-20800, between the National Aeronautics and Space Administration (NASA) and the U.S. Department of Transportation, FAA. This work could not have been possible without the hard work, diligence, assistance and support of Mr. Harry Hodges, Mr. Doug Rodzon, and the entire AFS-440 Flight Simulation Branch. The support of Mr. Bruce DeCleene (AFS-400) was also critical.

The technical assistance of Dr. Robert "Buck" Joslin, the FAA Chief Scientist and Technical Advisor for Flight Deck Technology Integration, was critical to the simulation planning. Further, the help of Mr. Bryan Burks, Alaska Airlines, was instrumental in the development of our test scenarios. 


\section{REFERENCES}

1 Wilson, J., Harron, G., Lyall, B., Hoffa, B., and Jones, E. "Understanding Single Pilot Operations (SPO) Certification Requirements," Research Integrations, Inc., 1 Dec 2013

2 Norman, R.M., "Economic Opportunities and Technological Challenges for Reduced Crew Operations." NASA Contractor Report, NASA Contract, Contract NNL06AA04B, May 2007.

3 Harris, D., "A human-centered design agenda for the development of single crew operated commercial aircraft", Aircraft Engineering and Aerospace Technology, Vol. 79, Issue 5, 2007, pp. 518-526

4 Graham, J., Hopkins, C., Loeber, A., and Trivedi, S., "Design of a single pilot cockpit for airline operations." Systems and Information Engineering Design Symposium (SIEDS), 2014. IEEE, 2014.

5 Cummings, M., Stimpson, A., and Clamann, M.. "Commercial Pilot Views on Futuristic Single Pilot Operations." Proceedings of the AIAA Aerospace Conference. 2016.

6 Li, G., Baker, S.P., Grabowski, J.G., and Rebok, G.W., "Factors Associated with Pilot Error in Aviation Crashes," Aviation, Space, and Environmental Medicine, Vol. 72, No. 1, January 2001

7 Federal Aviation Administration Advisory Circular: Aviation Safety Action Program, AC-120-66B, Dated November, 2002.

8 Emergency and Abnormal Situations in Aviation Symposium, LOSA Data and Emergency and Abnormal Situations http://humanfactors.arc.nasa.gov/flightcognition/download/EAS_Sy mposium_Presentations/LOSA.pdf , June 10-11, 2003

9 Air Line Pilots Association, "Air Line Pilots Association White Paper on Unmanned Aircraft Systems," April 2011

10 Fischer, M.R. "System and process for providing improved aircraft operational safety." U.S. Patent No. 7,271,740. 18 Sep. 2007

11 Jones, R.D., Whelan, D.A., and Wenberg, L.L,. "Variably manned aircraft." U.S. Patent No. 8,255,098. 28 Aug. 2012

12 Levine, S. and Levine, L.J.L., "An Onboard Pilot And Remote Copilot For Aviation Safety, Security \& Cost Savings." Digital Avionics Systems Conference, 2007. DASC'07. IEEE/AIAA 26th. IEEE, 2007

13 Defense Advanced Research Projects Agency, "Aircrew Labor InCockpit Automation System," retrieved at http://www.darpa.mil/program/aircrew-labor-in-cockpitautomation-system, July 2017

14 Bayram, K., et al, "Advanced Cockpit for Reduction Of Stress and Workload, Scope and State of the Art," Deutsches Zentrum für Luft- und Raumfahrt; DLR, Document Number: ACROSS/WP1/DLR/TECH/DEL/002, June 2013

15 Lachter, J., Brandt, S.L., Battiste, V., Ligda, S.V., Matessa, M., and Johnson, W.W., "Toward Single Pilot Operations: Developing a Ground Station" Proceedings of the International Conference on Human-Computer Interaction in Aerospace. ACM, 2014

16 Brandt, S.L., Lachter, J., Battiste, V., and Johnson, W.W., "Pilot situation awareness and its implications for single pilot operations: analysis of a human-in-the-loop study." Procedia Manufacturing, Volume 3, 2015, Pages 3017-3024.

17 Casner, S.M., "The Pilot-into-the-Loop Problem: Joining and Rejoining Flight in Progress." International Journal of Applied Aviation Studies, Volume 10, Issue 2, Dec 2010, pp. 1-9

18 Lachter, J., Battiste, V., Matessa, M., Dao, Q.V., Koteskey, R., and Johnson, W.W., "Toward Single Pilot Operations: The Impact of the Loss of Non-verbal Communication on the Flight Deck." Proceedings of the International Conference on Human-Computer Interaction in Aerospace. ACM, 2014
19 Xiao, Y., and Mackenzie, C.F., "Collaboration in Complex Medical Systems." In RTO-MP-4 AC/323(HFM)TP/2, Collaborative Crew Performance in Complex Operational Systems, RTO Human Factors and Medicine Panel (HFM) Symposium, Edinburgh, United Kingdom, 20-22 April 1998.

20 Flight Safety Foundation, "A Practical Guide for Improving Flight Path Monitoring. Flight Safety Foundation," Final Report of The Active Pilot Monitoring Working Group. Nov 2014

21 DeJohn, C.A., Wolbrink, A.M., and Larcher, J.G., "In-Flight Medical Incapacitation and Impairment of U.S. Airline Pilots: 1993 to 1998," FAA Report No. DOT/FAA/AM-04/16, Oct 2004.

22 Mitchell, SJ., and Evans, A.D., "Flight safety and medical incapacitation risk of airline pilots." Aviation, space, and environmental medicine. Vol. 75, Issue 3 (2004): pp. 260-268.

23 Evans, S. and Radcliffe, S-A., "The annual incapacitation rate of commercial pilots." Aviation, space, and environmental medicine Vol. 83, Issue 1 (2012): pp. 42-49.

24 Harrivel, A., et al, "Prediction of cognitive states during flight simulation using multimodal psychophysiological sensing." Paper presented at AIAA Infotech @ Aerospace, AIAA paper 2017-1135, Jan. 2017.

25 Caldwell, J.A.; Mallis, M.M.; Caldwell, J.L., Paul, M.A., Miller, J.C., and Neri, D.F., "Fatigue Countermeasures in Aviation," Aviation, Space, and Environmental Medicine, Volume 80, Number 1, January 2009, pp. 29-59.

26 Civil Aviation Authority, "A Review of In-flight Napping Strategies" CAA Paper 2003/8, 1 Sept 2003.

27 Simons, M., and Spencer, M., "Extension of flying duty period by inflight relief." TNO Report TNO-DV 2007 C362, Sept 2007.

28 National Research Council (NRC). "Autonomy Research for Civil Aviation: Toward a New Era of Flight," Final Report from the "Committee on Autonomy Research for Civil Aviation," Aeronautics and Space Engineering Board, Division on Engineering and Physical Sciences, 2014.

29 Etherington, T.J., Kramer, L.J., Bailey, R.E., Kennedy, K.D., and Stephens, C.L., Quantifying Pilot Contribution to Flight Safety For Normal And Non-Normal Airline Operations. 2016 IEEE/AIAA 35th Digital Avionics Systems Conference (DASC), Sacramento, CA, USA, 25-29 Sept 2016.

30 Etherington, T.J., Kramer, L.J., Bailey, R.E., and Kennedy, K.D., Quantifying Pilot Contribution to Flight Safety During An In-Flight Airspeed Failure. 19th International Symposium on Aviation Psychology, Wright State University, Dayton, Ohio, USA, 8 - 11 May 2017

31 Kramer, L.J., Etherington, T.J., Bailey, R.E., and Kennedy, K.D., Quantifying Pilot Contribution to Flight Safety during Hydraulic Systems Failure. 8th International Conference on Applied Human Factors and Ergonomics (AHFE 2017), Los Angeles, California, USA, 17-21 July 2017.

32 Kramer, L.J., Etherington, T.J., Bailey, R.E., and Kennedy, K.D., "Quantifying Pilot Contribution to Flight Safety during Drive Shaft Failure.” 2017 IEEE/AIAA 36th Digital Avionics Systems Conference (DASC), St. Petersburg, FL, USA, 17-21 Sept 2017.

33 Etherington, T.J., Kramer, L.J., Bailey, R.E., and Kennedy, K.D., "Quantifying Pilot Contribution to Flight Safety during Dual Generator Failure." 2017 IEEE/AIAA 36th Digital Avionics Systems Conference (DASC), St. Petersburg, FL, USA, 17-21 Sept 2017.

34 Hart, S.G. and Staveland, L.E., "Development of NASA-TLX (Task Load Index): Results of Empirical and Theoretical Research.” In P.A. 
Hancock \& N. Meshkati (Eds.), Human Mental Workload. Amsterdam: North-Holland, 1988, pp. 139-183.

35 Vidulich, M.A., "The Use of Judgment Matrices in Subjective Workload Assessment: The Subjective Workload Dominance (SWORD) Technique." Proceedings of Human Factors and Ergonomics Society, Vol. 33, Issue 20, 1989

36 Hindson, W.S., Eshow, M.M., and Schroeder, J.A., "A Pilot Rating Scale For Evaluating Failure Transients In Electronic Flight Control Systems," paper presented at AIAA Atmospheric Flight Mechanics Conference, Portland, Oregon, AIAA Paper No. 1990-2827, Aug 1990.
37 Mumaw, R. and Feary, M., "Aircraft Capability Management." Presentation at the NextGen Flight Deck and Human-Autonomy Teaming Symposium, NASA Langley Research Center, Feb 2017.

38 Mehta, S.S., Doucette, E.A., and Curtis, J.W., "Curious partner: an autonomous system that proactively dialogues with human teammates." Proceedings of SPIE Vol. 10194, Micro- and Nanotechnology Sensors, Systems, and Applications IX, May 2017.

39. Cummings, M.L., Stimpson, A., and Clamann, M., "Functional Requirements for Onboard Intelligent Automation in Single Pilot Operations." AIAA Infotech @ Aerospace, AIAA Paper No. 20161652, San Diego, CA, Jan. 2016. 